\title{
ORIGEM DO POTENCIAL ELÉTRICO ESPONTÂNEO EM ROCHAS CRISTALINAS FRATURADAS E SUA UTILIZAÇÃO NA LOCAÇÃO DE POÇOS
}

\author{
W. E. de Medeiros ${ }^{1}$ \& O. A. L. de Lima ${ }^{2}$
}

Medidas de potencial elétrico espontâneo (PE) foram realizadas no interior de poços e na superfície da terra, ao longo de seções transversais, em aqüíferos fissurais em rochas cristalinas do Estado da Bahia. Estas medidas revelam a presença de fortes anomalias de PE relacionadas à movimentação da água subterrânea. Com base em um modelo geométrico-hidráulico simplificado para um aqüífero freático, numa estrutura essencialmente bi-dimensional, e usando as equações básicas para os acoplamentos de fluxos de fluidos (ou de calor) e de concentração iônica, é mostrado que os efeitos eletrocinéticos desenvolvidos ao longo das paredes das fraturas podem produzir anomalias de $\mathrm{PE}$ comparáveis àquelas observadas no campo. Por outro lado, as estimativas obtidas para os acoplamentos associados aos efeitos eletroquímico e termo-elétrico são bem menores do que os estimados para o efeito eletrocinético, o que sugere que este último constitui a fonte dominante das anomalias de PE observadas em zonas fraturadas de rochas cristalinas. Com base neste resultado, foi feita a interpretação integrada de seções de resistividade aparente e perfis de PE de campo. Nesta interpretação, ficou evidenciado que o método de PE pode ser usado como uma técnica geofísica complementar ao método de eletro-resistividade na distinção de áreas com permeabilidade relativamente alta no interior de aqüíferos com porosidade fissural.

Palavras-chave: Água subterrânea; Potencial espontâneo; Aqüiferos fissurais.

SELF-POTENTIAL ANOMALY GENERATION IN FRACTURED CRYSTALLINE ROCKS AND ITS USE TO BOREHOLE LOCATION-Self-potential electric (SP) measurements made in wells and at the surface along traverses crossing aquifers in fractured crystalline rocks in the state of Bahia, Brazil, revealed the presence of strong anomalies related to groundwater movements. By considering a simple geometrichydraulic model for a phreatic aquifer, in an essentially 2-D structure, and using basic equations for ionic concentration and fluid (or heat) coupled flows, it was found that electrokinetic effects developed along fracture walls can produce anomalies of amplitude comparable to those observed in the field. Estimates obtained for other coupled processes, such as the electrochemical and the thermo-electric effects, suggest that the electrokinetic effect is the most important component for setting up SP currents in fractured zones of crystalline rocks. Based on this result, a joint interpretation of pseudoresistivity sections and SP profiles was performed and the results show that the SP method can be used as a complementary geophysical technique to the resistivity method, allowing to distinguish areas of relative high permeability within fractured rocks.

Key words: Groundwater; Self-Potential; Fractured rocks.

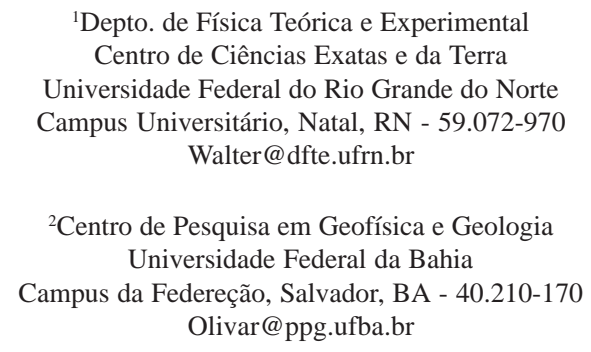

Brazilian Journal of Geophysics, Vol. 17(2,3), 1999 


\section{INTRODUÇÃO}

O método do Potencial Espontâneo (PE) constitui uma das mais antigas técnicas de prospecção geofísica e tem continuamente sido utilizado, com sucesso, numa grande variedade de problemas de hidrogeologia, geotecnia e prospecção. Como exemplos, citamos a prospecção de água subterrânea em formações sedimentares (Schiavone \& Quarto, 1984), a localização de zonas de perda d'água em grandes barragens (Olgivy et al., 1969), a avaliação de fontes geotermais (Anderson \& Johnson, 1976; Corwin \& Hoover, 1979; Corwin et al., 1981), a prospecção de depósitos de sulfetos metálicos (Sato \& Money, 1960; Logn \& Bolviken, 1974; Corry, 1985), as pesquisas arqueológicas (Wynn \& Sherwood, 1984), e as tentativas de previsão de terremotos (Mizutani et al., 1976; Corwin \& Morrison, 1977; Di Maio \& Patella, 1991) e erupções vulcânicas (Di Maio \& Patella, 1994; Di Maio et al., 1996). Uma recente compilação sobre as aplicações de PE pode ser encontrada em Patella (1997).

A origem das anomalias de PE no meio geológico tem sido atribuída, basicamente, a fenômenos eletroquímicos, termo-elétricos e eletrocinéticos (Corwin \& Hoover, 1979). Essa diversidade de fontes causativas permite uma extensiva aplicação prática mas, em contrapartida, traz grande dificuldade para identificar o mecanismo de geração dominante em cada caso.

A necessidade de otimizar as técnicas de locação de poços em aqüíferos fissurais nas rochas cristalinas do Nordeste do Brasil tem despertado o interesse de vários pesquisadores. Neste sentido, Lima \& Medeiros (1988) e Medeiros \& Lima (1990) constataram, em um estudo geo-elétrico com vistas à identificação das zonas produtoras de água, que as zonas de entrada de água nos poços estão correlacionadas com fortes anomalias de PE (superiores a $100 \mathrm{mV}$ ). Posteriormente, os mesmos autores (Medeiros \& Lima, 1991) efetuaram medições de PE ao longo de perfis na superfície do terreno em Ipirá (BA) e constataram a presença de anomalias com amplitude de até $60 \mathrm{mV}$, associadas às areas mais intensamente fraturadas do embasamento cristalino.

O presente trabalho tem como objetivo efetuar uma análise dos possíveis mecanismos geradores de correntes de PE em rochas cristalinas fraturadas, de modo a identificar as fontes dominantes para as anomalias observadas e destacar as informações hidrogeológicas que podem ser extraídas dessa análise. De modo a fornecer subsídios para a análise, o tra- balho inicia com uma revisão sobre os mecanismos geradores de PE nas rochas e sobre a teoria básica de fluxos acoplados, que é utilizada no cálculo de anomalias teóricas de PE (Sill, 1983). A seguir, usando um modelo geométrico e hidráulico simplificado para os aquífferos fissurais em rochas cristalinas, são estimadas as ordens de grandezas das anomalias de PE associadas aos efeitos eletrocinético, termo-elétrico e eletroquímico, permitindo assim a identificação do primeiro como sendo o mecanismo dominante. Por fim, são apresentados e discutidos dados experimentais de campo com o objetivo de evidenciar a importante contribuição que o método de PE pode fornecer na locação de poços produtores de água em aqüíferos cristalinos.

\section{GERAÇÃO DE POTENCIAL ESPONTÂNEO NAS ROCHAS}

\section{Efeito Eletrocinético}

Considere uma amostra de rocha porosa saturada com um fluido salino. Se as extremidades da amostra forem submetidas a um diferença de pressão, $\Delta \mathrm{p}$, de modo a produzir um fluxo hidráulico, q, é também produzida uma diferença de potencial elétrico (d.d.p.), $\Delta \Phi$, dada por:

$$
\Delta \Phi=\mathrm{C} \Delta \mathrm{p}
$$

em que C é o coeficiente de acoplamento eletrocinético (Dakhnov, 1962).

A origem deste fenômeno está associada com a formação de uma dupla camada de cargas elétricas nas paredes dos poros da rocha, comumente denominada de dupla camada de Helmholtz. Esta dupla camada se forma porque a superfície dos poros não está eletricamente balanceada (geralmente em conseqüência de trocas iônicas) e adsorve íons de sinal contrário dissolvidos no fluido de saturação. Forma-se, então, no fluido, uma camada de íons, parte imóvel e parte difusa. A parte difusa pode se movimentar se o fluido for submetido a uma diferença de pressão, gerando assim uma corrente elétrica por convecção.

Para tubos capilares, é possível determinar analiticamente o valor do coeficiente C (Daknhov, 1962). Contudo, para as rochas, esta determinação é muito difícil face a complexidade dos fatores envolvidos. Por outro lado, medições de laboratório em amostras de mão evidenciam que $\mathrm{C}$ pode variar de 1 a $70 \mathrm{mV} / \mathrm{atm}$ (Ahmad, 1964; Olgivy et al., 1969), sendo usualmente 
maior para rochas de porosidade intersticial (Fitterman, 1978). Medidas in situ de C não são conhecidas.

Em rochas cristalinas, o coeficiente $\mathrm{C}$ tem sido pouco analisado, certamente devido às dificuldades de se obter amostras representativas de rochas fraturadas que, usualmente, exigem um volume de amostragem da ordem de metros cúbicos. Para contornar esta dificuldade, Bogoslovsky \& Olgivy (1972) simularam sistemas de fraturas com placas de vidro e estudaram a influência sobre $\mathrm{C}$ da abertura das fissuras, da salinidade da água de saturação e da quantidade e constituição do material granular de preenchimento das fissuras. Em linhas gerais, eles concluíram que $\mathrm{C}$ é inversamente proporcional à abertura das fissuras e à salinidade da água, e diretamente proporcional à quantidade de areia de quartzo existente nas fraturas. Em relação à salinidade, foi indicado o limiar de $0,1 \Omega \mathrm{m}$ para a resistividade da água de saturação, abaixo do qual nenhuma d.d.p. poderia ser medida.

As águas do cristalino do Nordeste Brasileiro têm resistividades de cerca de $5 \Omega \mathrm{m}$. De acordo com as conclusões de Bogoslovsky \& Olgivy (1972), este valor é grande o suficiente para gerar anomalias de PE por efeito eletrocinético. Com águas mais salinas, Tuman (1963, apud Corwin \& Hoover, 1979) mediu, em arenitos, valores de $\mathrm{C}$ de cerca de $15 \mathrm{mV} / \mathrm{atm}$.

\section{Efeito Termo-elétrico}

Quando uma amostra de rocha é submetida a uma diferença de temperatura, $\Delta \mathrm{T}$, é gerada entre as suas extremidades uma d.d.p. Esta d.d.p. é causada pela difusão térmica diferencial dos íons na solução, bem como pela difusão de elétrons na matriz da rocha (Corwin \& Hoover, 1979). Uma equação análoga à Eq. (1) pode ser proposta (com $\Delta \mathrm{T}$ substituindo $\Delta \mathrm{p}$ ), sendo o coeficiente de acoplamento termo-elétrico da ordem de $0,2 \mathrm{mV} /{ }^{\circ} \mathrm{C}$ para rochas (Corwin \& Hoover, 1979).

Vários autores já propuseram que o efeito termoelétrico é o principal causador das anomalias de PE observadas em fontes geotermais onde atuam elevados gradientes geotérmicos (e.g., Anderson \& Johnson, 1976; Corwin \& Hoover, 1979).

\section{Efeito Eletroquímico}

Quando águas de diferentes concentrações salinas são mantidas em contato, íons se difundem da solução mais concentrada para a solução mais diluída. Devido às diferenças de mobilidade entre os íons, uma corrente elétrica é gerada (Daknhov, 1962). Além disso, se existir uma camada de argila separando as diferentes soluções, preferencialmente os cátions podem atravessá-la. Este fenômeno ocorre porque na estrutura interna dos minerais de argila existem camadas com excesso de cargas positivas que adsorvem os ânions (Keller \& Frischknecht, 1966). Estes dois efeitos combinados geram uma d.d.p. dada por (Dakhnov, 1962):

$$
\Delta \phi=-\mathrm{K} \log _{10}\left(\frac{R^{-}}{R^{+}}\right),
$$

em que $\mathrm{R}^{-}$e $\mathrm{R}^{+}$são as resistividades das soluções de menor e maior concentração salina, respectivamente, e K é um parâmetro (com dimensão de potencial elétrico) que depende da natureza e temperatura do eletrólito. Por exemplo, para cloreto de sódio e temperaturas de cerca de $30^{\circ} \mathrm{C}, \mathrm{K} \approx 70 \mathrm{mV}$.

\section{Equações de fluxos acoplados}

O acoplamento generalizado de fluxos de concentração e energia pode ser descrito por (Marshall \& Madden, 1959; Fitterman, 1978; Sill, 1983):

$$
\vec{\Gamma}_{i}=-\sum_{j} L_{i j} \vec{\nabla} \Phi j,
$$

em que $\stackrel{r}{\Gamma}_{i}$ são os fluxos (de carga, massa, calor, etc), $\Phi_{\mathrm{j}}$ são os potenciais (elétrico, de pressão, de temperatura, etc) e $L_{\mathrm{ij}}$ são as condutividades generalizadas.

Para o caso do acoplamento eletrocinético, por exemplo, a Eq. (3) se reduz a

$$
\left(\begin{array}{l}
\vec{J} \\
\vec{q}
\end{array}\right)=-\left(\begin{array}{cc}
\sigma & L_{12} \\
L_{21} & k
\end{array}\right)\left(\begin{array}{l}
\vec{\nabla} \phi \\
\vec{\nabla} p
\end{array}\right),
$$

em que $\vec{J}$ é a densidade de corrente elétrica, $\vec{q}$ é a velocidade de filtração ou vazão específica, $\sigma$ é a condutividade elétrica, $\mathrm{k}$ é a permeabilidade hidráulica, $\mathrm{L}_{12} / \sigma=\mathrm{C}$ é o coeficiente de acoplamento eletrocinético, $\mathrm{L}_{21} / \mathrm{k}$ é o coeficiente de acoplamento eletro-osmótico, $\Phi$ é o potencial elétrico e $p$ é a pressão. A relação de reciprocidade de Onsager requer que $L_{12}=L_{21}=L$ (Marshall \& Madden, 1959). Da Eq. (4) obtém-se:

$$
\begin{gathered}
\vec{j}=-\sigma \vec{\nabla} \phi-L \vec{\nabla} p, \\
\vec{q}=-k \vec{\nabla} p-L \vec{\nabla} \phi,
\end{gathered}
$$

$\mathrm{Na}$ ausência de fontes elétricas externas e no estado estacionário, $\vec{\nabla} \vec{j}=0$. Assim, em regiões 
homogêneas de $\sigma$ e $L$, obtém-se da Eq. (5) que

$$
\sigma \nabla^{2} \Phi+L \nabla^{2} p=0
$$

Aplicando o operador divergente na Eq. (6) e usando a Eq. (7) obtém-se, no estado estacionário $(\vec{\nabla} \cdot \vec{q}=0)$ e em regiões onde $k$ e $L$ são constantes,

$$
\vec{\nabla} \cdot \vec{q}=-k\left[1-L^{2} /(\sigma k)\right] \nabla^{2} p \approx-k \nabla^{2} p=0,
$$

porque, para rochas, $\mathrm{L}^{2} /(\sigma \mathrm{k}) \approx 0,001<1$ (Fitterman, 1978). Em consequiência, a Eq. (6) pode ser desacoplada da Eq. (5) e resolvida sem levar em conta o efeito do potencial elétrico. Observe que esta aproximação implica desprezar o efeito elétrico sobre o efeito hidráulico mas não o contrário. De agora em diante, sempre que for citada a Eq. (6) estará implícita esta aproximação de desprezar o acoplamento eletroosmótico. Desse modo, $p$ pode ser chamado de potencial primário e $\Phi$, de potencial secundário. Uma aproximação análoga pode ser feita para o caso do acoplamento termo-elétrico, onde a equação do fluxo de calor é desacoplada e a temperatura torna-se o potencial primário (Sill, 1983).

Para resolver as Eqs. (5) e (6), a abordagem de Sill (1983) pode ser seguida. Nesta abordagem, os potenciais primário e secundário são separados e a densidade de corrente elétrica $\vec{J}$, na Eq. (5) pode ser escrita como

$$
\stackrel{\mu}{J}=\stackrel{\mu}{J}_{\text {cond }}+\stackrel{\mu}{J}_{\text {conv }},
$$

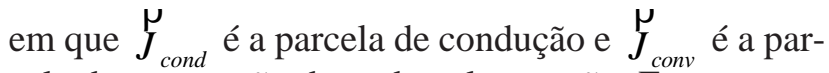
cela de convecção dragada pela pressão. Estas parcelas são definidas por:

$$
\begin{aligned}
& \stackrel{\mu}{J} \text { cond }=-\sigma \vec{\nabla} \phi, \\
& J_{\text {conv }}=-L \vec{\nabla} \mathrm{p} .
\end{aligned}
$$

$\mathrm{Na}$ ausência de fontes ou de sorvedouros (tanto elétricos quanto hidráulicos), os termos de fonte estão necessariamente associados com variações dos parâmetros físicos, como é mostrado a seguir. A conservação da carga elétrica implica que

$$
\vec{\nabla} \cdot \vec{J}_{\text {cond }}=-\vec{\nabla} \cdot \vec{J}_{\text {conv }}=\vec{\nabla} L \cdot \vec{\nabla} p+L \nabla^{2} p
$$

Portanto, existem fontes de corrente elétrica quando há um gradiente de pressão normal a uma superfície de descontinuidade em $L$ e/ou quando existem fontes externas ou induzidas de pressão. Uma expressão mais conveniente para o termo $L \nabla^{2} p$ pode ser obtida aplicando-se o operador divergente na Eq. (6) (desprezando o termo de acoplamento eletro-osmótico) e considerando o estado estacionário de fluxo da água subterânea (i.e., $\vec{\nabla} \vec{q}=0$ ), como mostrado a seguir:

$$
\vec{\nabla} \cdot \vec{q}=-\vec{\nabla} k \cdot \vec{\nabla} p-k \nabla^{2} p=0
$$

$\log 0$

$$
L \nabla^{2} p=-\frac{L}{k} \vec{\nabla} k \cdot \vec{\nabla} p
$$

Na Eq. (14) o termo $\frac{L}{k} \vec{\nabla} \cdot \vec{q}$ só é diferente de zero quando existem fontes ou sorvedouros de fluido, e o termo $\frac{L}{k} \vec{\nabla} k \cdot \vec{\nabla} p$ atua como uma fonte elétrica secundária devido à pressão. Na ausência de extração ou injeção de água em um aquiífero e combinando-se as Eqs. (11), (12) e (14), pode-se obter:

$$
\vec{\nabla} \cdot \vec{J}_{\text {cond }}=\vec{\nabla} L \cdot \vec{\nabla} p-\frac{L}{k} \vec{\nabla} k \cdot \vec{\nabla} p
$$

Desse modo, para determinar o potencial elétrico espontâneo, $\Phi$, devido a uma dada estrutura, é necessário primeiramente resolver a Eq. (8) para, então, introduzir os termos de fonte na Eq. (15) e resolvê-la.

O desenvolvimento acima apresentado é também válido para os acoplamentos termo-elétrico e eletroquímico, uma vez realizadas as devidas substituições das condutividades generalizadas.

\section{MECANISMO DOMINANTE EM AQÜIFEROS FISSURAIS}

A Eq. (15) pode ser numericamente resolvida com o uso da técnica de diferenças finitas ou de elementos finitos, adaptando-se um algoritmo de cálculo do potencial elétrico em corrente direta. Em aquiíferos fissurais em rochas cristalinas, por exemplo, é suficiente resolver o problema na seção transversal ao eixo principal de fraturamento, de modo que a abordagem de modelamento é bidimensional (Medeiros, 1987), como será melhor discutido na seção a seguir. Contudo, em qualquer caso de acoplamento, é necessário a caracterização completa da seção transversal do aquífero. Por exemplo, para o caso eletrocinético é preciso dispor da descrição nesta seção das variáveis: pressão $(p)$, permeabilidade $(\mathrm{k})$, condutividade elétrica $(\sigma)$ e coeficiente de acoplamento eletrocinético $(C$ ou $L=C \sigma)$. Para aqüíferos fissurais no cristalino do Nor- 
deste do Brasil, não se dispõe de uma descrição com tal nível de detalhe e, assim, deve-se evitar a sofisticação de fornecer uma resposta numericamente precisa com base em dados imprecisos. Neste trabalho, utilizou-se a Eq. (15), em associação com um modelo físico simples e realista para aqüíferos fissurais, visando estimar as ordens de grandeza do PE gerado pelos vários tipos de acoplamento. Assim, pode-se identificar qual é o mecanismo dominante de geração das anomalias de PE neste tipo de aquífero.

\section{Efeito Eletrocinético}

Os aqüíferos das rochas cristalinas do Nordeste Brasileiro têm porosidade definida por superfícies de rupturas, por onde é possível ocorrer infiltração, armazenamento e migração da água subterrânea; tais descontinuidades, por se constituirem em zonas de mais fácil erosão das rochas cristalinas que constituem os terrenos, condicionam freqüentemente a formação de linhas de drenagem superficial, formando os conhecidos "riachos-fenda" da comunidade hidrogeológica do Nordeste Brasileiro. O grupo de fraturas define conjuntos de planos, paralelos ou interceptados entre si, resultando que as direções dos fluxos subterrâneos são comandadas por este padrão. Em geral, as direções vertical e horizontal são preponderantes, o que permite efetuar uma análise bidimensional considerando apenas o plano transversal à direção do riacho-fenda.

A Fig. 1 apresenta uma seção transversal esquemática do modelo genérico de aquífero válido para as rochas cristalinas do Nordeste Brasileiro. Esta seção resulta de uma série de investigações geofísicas realizadas em aquíferos fissurais em rochas cristalinas (Medeiros, 1987; Lima \& Medeiros, 1988; Medeiros \& Lima, 1990, 1991). Da base para o topo, são descritas a seguir as unidades que compõem o modelo da Fig. 1: (i) a unidade 5 é a rocha cristalina sã, quase sem fraturas ou com as fraturas fechadas pela pressão de confinamento; (ii) a unidade 4 é a rocha cristalina fraturada; (iii) as unidades 3 e 2 constituem as zonas de alteração mais avançadas da rocha cristalina, sendo a unidade 2 de constituição mais arenosa do que a unidade 3 , devido à maior lixiviação proporcionada pela proximidade do riacho; e (iv) a unidade 1 é o solo acima da superfície freática. As unidades 2 e 4 são as principais estruturas de armazenamento e percolação de água, apresentando a unidade 2 porosidade granular e a unidade 4 porosidade fissural.

Com base na constituição do material, tipo e grau de fraturamento, e resultados experimentais da literatura, propõe-se para as várias unidades os valores de
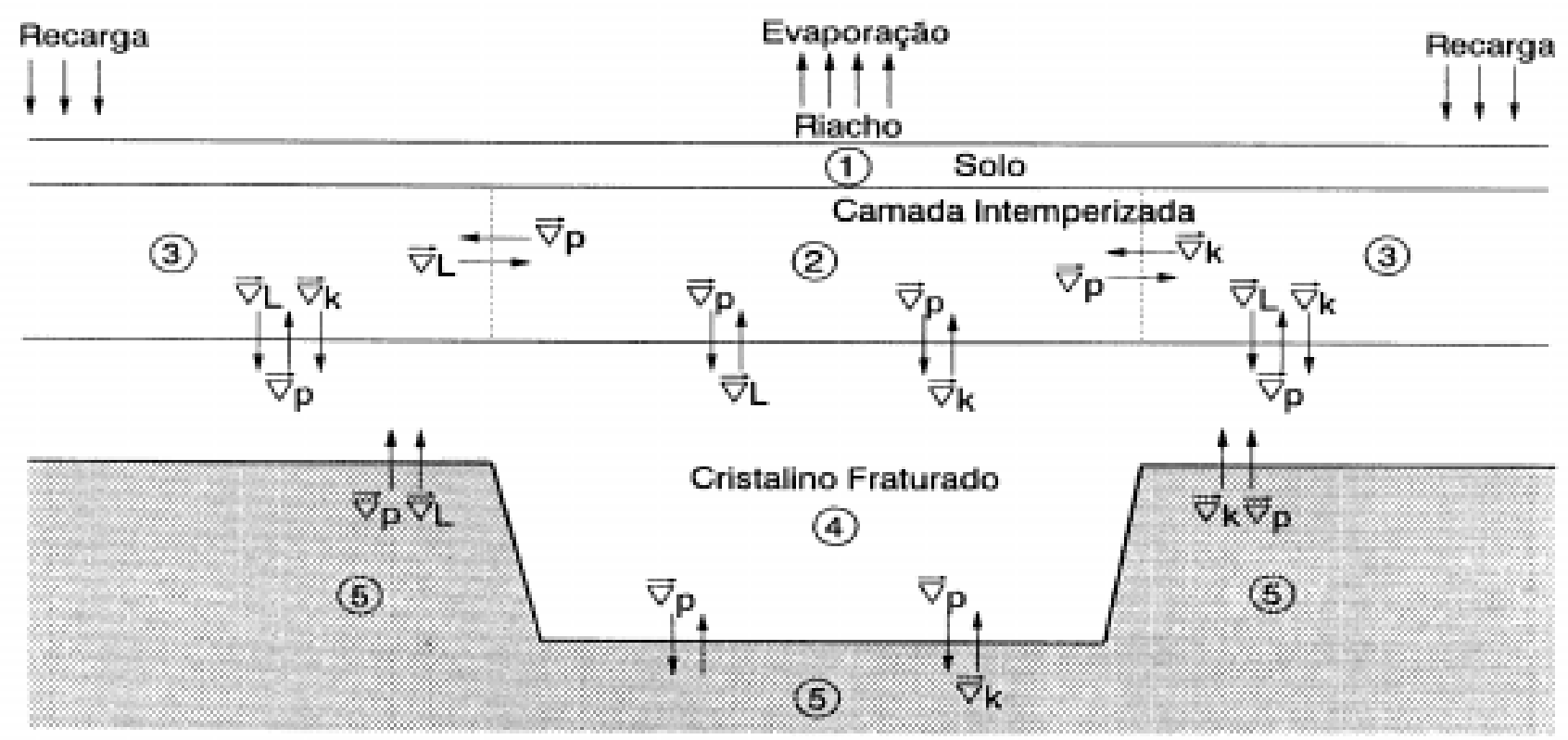

Figura 1 - Modelo geométrico-hidráulico para um aqüífero fissural em rochas cristalinas $\vec{\nabla} p, \vec{\nabla} \mathrm{k}$ e $\vec{\nabla} \mathrm{L}$ são os gradientes de pressão, de permeabilidade e de coeficiente de acoplamento eletrocinético, respectivamente. Unidade 1: solo. Unidades 2 e 3: zonas de alteração da rocha cristalina. Unidade 4: rocha cristalina fraturada. Unidade 5: rocha cristalina sã.

Figure 1 - Geometric-hydraulic model for fractured aquifers in crystalline rocks $\vec{\nabla} p, \vec{\nabla} \mathrm{k}$ e $\vec{\nabla} \mathrm{L}$ are gradients of pressure, permeability, and electrokinetic coefficient, respectively. Unity 1: soil. Unities 2 and 3: weathering products. Unity 4: fractured crystalline rock. Unity 5: fresh crystalline rock. 


$\begin{array}{cccc}\text { Unidade } & \begin{array}{c}\sigma \\ (\Omega \mathrm{m})^{-1}\end{array} & \begin{array}{c}\mathrm{C} \\ \mathrm{mV} / \mathrm{atm}\end{array} & \begin{array}{c}\mathrm{k} \\ \mathrm{cm} / \mathrm{s}\end{array} \\ 2 & 10^{-1} & 15 & 10^{-2} \\ 3 & 10^{-1} & 1 & 10^{-5} \\ 4 & 10^{-2} & 5 & 10^{-3} \\ 5 & \infty & 0 & 10^{-6}\end{array}$

Tabela 1- Valores típicos de condutividade elétrica $(\sigma)$, coeficiente de acoplamento eletrocinético $(C)$ e permeabilidade (k) para as unidades do modelo de aquíf́ero cristalino mostrado na Fig. 1.

Table 1- Typical values for electrical conductivity $(\sigma)$, electrokinetic coefficient $(C)$, and permeability $(k)$ for the unities of the fractured aquifer model shown in Fig. 1.

propriedades físicas mostrados na Tab. 1. É atribuída uma maior confiança aos valores de condutividade elétrica porque eles são originados da interpretação de sondagens e seções de campo de resistividade aparente. Os valores de permeabilidade e coeficiente de acoplamento eletrocinético foram coletados na literatura (Bogoslovsky \& Olgivy, 1972; Benedini, 1976; Corwin \& Hoover, 1979), levando-se em conta as condições de porosidade e de salinidade típicas dos aqüíferos fissurais em rochas cristalinas do Nordeste Brasileiro. A unidade 1 não consta da Tab. 1 por apresentar valores muito variáveis. Além disso, ela tem pouca influência no processo.

Para especificar os termos de fonte da Eq. (15) é necessário também dispor de um modelo hidrodinâmico para o aquíf́ero. Uma boa aproximação é a de aqüífero livre, pois o modelo genérico mostrado na Fig. 1 constitui-se basicamente de camadas permeáveis superpostas ao embasamento cristalino impermeável, sem apresentar nenhum estrato confinante acima deste embasamento; tais camadas estão saturadas e o seu limite superior é o lençol freático local, portanto submetido apenas à pressão atmosférica. Nesta situação, o fluxo da água subterrânea é controlado basicamente pela topografia. Estas condições são particularmente válidas para a estação não chuvosa, ocasião em que o riacho está seco e não há alimentação do aqüífero a partir do aluvião do riacho. Os dados de PE que serão apresentados na próxima seção foram coletados durante estações não chuvosas.

O movimento da água subterrânea é, assim, basicamente sub-horizontal e convergente para o vale do riacho. Devido às condições de simetria em relação ao vale, este deve se constituir num ponto de estagnação da velocidade da água na direção horizontal. Além disso, o movimento tem uma componente vertical para baixo na zona de recarga (que são os altos topográficos) e uma componente vertical para cima na zona de des- carga (que são os baixos topográficos, particularmente, o vale do riacho), variando continuamente de um extremo ao outro. Existe também uma componente na direção perpendicular ao plano da Fig. 1, no sentido montantejusante do riacho, que não será considerada neste estudo. Eventualmente, a depender da interconectividade da trama de fraturas, pode haver confinamento local.

Usando um gradiente médio de pressão de 5\% (i.e., o nível natural da água baixando de $5 \mathrm{~cm}$ numa dada direção para cada metro de distância na direção transversal) e os dados da Tab. 1, pode-se estimar as ordens de grandeza dos termos de fonte da Eq. (15), associados às várias interfaces entre as unidades do aqüífero. A convenção de sinal implícita na definição da Eq. (15) significa que, ao atravessar uma unidade no mesmo sentido de $\vec{\nabla} p$, a densidade de corrente é positiva se $\vec{\nabla} L \cdot \vec{\nabla} p>0$ (ou seja, se $\vec{\nabla} L$ tem o mesmo sentido de $\vec{\nabla} p$ ) e se $\vec{\nabla} k \cdot \vec{\nabla} p<0$ (ou seja, se $\vec{\nabla} k$ tem sentido contrário a $\vec{\nabla} p)$.

Seja $\delta$ a distância típica em que ocorrem as variações espaciais de propriedade física nestas interfaces. A densidade de corrente por unidade de comprimento $\left(J_{1} / \delta\right)$, induzida na superfície de separação entre as unidades 2 e 3 (Fig. 1) e associada ao termo $\vec{\nabla} L \cdot \vec{\nabla} p$, por exemplo, pode ser estimada como

$$
\frac{J_{1}}{\delta}=\vec{\nabla} L \cdot \vec{\nabla} p=-|\vec{\nabla}(\sigma C) \cdot \vec{\nabla} p| \approx-5 \times 10^{-3} \mathrm{~mA} / \mathrm{m}^{3},
$$

pois,

$$
\begin{gathered}
|\vec{\nabla} p| \approx\left(5 \times 10^{-2} \mathrm{~m} / \mathrm{m}^{2}\right)\left(1 \times 10^{3} \mathrm{~kg} / \mathrm{m}^{3}\right)\left(10 \mathrm{~m} / \mathrm{s}^{2}\right) \\
\left(10^{-5} \mathrm{~Pa} / \mathrm{atm}\right) \approx 5 \times 10^{-3} \mathrm{~atm} / \mathrm{m}, \\
|\vec{\nabla} \mathrm{L}| \approx 0,1(15-1) \mathrm{mV} / \mathrm{atm} \approx 1 \mathrm{mV} /(\mathrm{atm} \mathrm{m}) .
\end{gathered}
$$

Na mesma interface entre as unidades 2 e 3, o termo de fonte associado à parcela $-L / k \vec{\nabla} k \cdot \vec{\nabla} p$ produz uma densidade de corrente por unidade de comprimento $\left(\mathrm{J}_{2} /\right.$ $\delta$ ) de cerca de $+7 \times 10^{-3} \mathrm{~mA} / \mathrm{m}^{3}$. Assim, a densidade de corrente total na interface entre as unidades 2 e 3 [que é igual a $\delta\left(\mathrm{J}_{1} / \delta+\mathrm{J}_{2} / \delta\right)$ ] é cerca de $+2 \times 10^{-3} \mathrm{~mA} / \mathrm{m}^{2}$ (Observe que a estimativa independe de $\delta$ ). De acordo com a convenção de sinal, o valor positivo significa que a corrente sai da unidade 2 e entra na unidade 3.

Procedendo de maneira análoga para a interface 
entre as unidades 2 e 4, obtém-se que a densidade de corrente total nesta interface é cerca de $+7 \times 10^{-3} \mathrm{~mA} /$ $\mathrm{m}^{2}$. Estimativas semelhantes podem ser obtidas para as demais interfaces.

O padrão geral das células de corrente elétrica geradas está esquematicamente mostrado na Fig. 2, juntamente com o perfil de PE gerado na superfície da Terra. O perfil de PE apresenta um mínimo com centro próximo ao riacho e lóbulos positivos, sendo a amplitude dos lóbulos bem menor que a amplitude do mínimo. Fora da região central, o perfil de PE apresenta um nível basicamente constante, pois a pressão (i.e., topografia) varia muito suavemente. A ordem de grandeza da amplitude do mínimo de PE pode ser estimada através da expressão genérica do potencial logarítmico para fontes de corrente bidimensionais que é dada por:

$$
\Delta \Phi=\gamma \frac{J \lambda}{\sigma} \ln \left(\frac{\lambda}{\lambda}\right)
$$

em que $\gamma$ é um parâmetro adimensional da ordem da unidade e que é função da geometria da seção transversal da fonte, $J$ é a densidade de corrente elétrica normal à superfície da fonte, $l_{0}$ é a distância a um ponto de referência convenientemente escolhido de modo a evitar a introdução de singularidades e $l$ é a distância a um ponto qualquer. Para pontos próximos da fonte, $\gamma \ln \left(\frac{\lambda}{\lambda_{0}}\right) \approx 1$. Além disso, para o modelo em questão, $\lambda_{0} \approx 10 \mathrm{~m}, \sigma \approx 10^{-2}(\Omega \mathrm{m})^{-1} \mathrm{e} \mathrm{J} \approx 10 \times 10^{-3} \mathrm{~mA} / \mathrm{m}^{2}$, de modo que $|\Delta \Phi| \approx 10 \mathrm{mV}$. Como será visto na próxima seção, o padrão geral das anomalias de PE de campo, inclusive os valores de amplitude, são bastante semelhantes aos obtidos neste modelo.

\section{Efeito Termo-elétrico}

Em áreas pré-cambrianas estáveis, a exemplo do cristalino do Nordeste Brasileiro, o gradiente vertical de temperatura é da ordem de $0,02^{\circ} \mathrm{C} / \mathrm{m}$. Utilizando o valor típico de coeficiente de acoplamento termoelétrico para rochas, que é igual a $0,2 \mathrm{mV} /{ }^{\circ} \mathrm{C}$ (Corwin \& Hoover, 1979), obtém-se que a parcela $\vec{\nabla} L \cdot \vec{\nabla} T$ [equivalente a $\vec{\nabla} L \cdot \vec{\nabla} p$ na Eq. (15)] gera uma densidade de corrente elétrica por unidade de comprimento de cerca de $4 \times 10^{-4} \mathrm{~mA} / \mathrm{m}^{3}$ nas interfaces horizontais. Esta densidade de corrente é uma ordem de grandeza menor que a gerada pelo efeito eletrocinético, o mesmo ocorrendo com a densidade de corrente associada com os demais termos equivalentes aos da Eq. (15). Nas interfaces verticais (Fig. 1), não é gerada praticamente nenhuma densidade de corrente elétrica, pelo fato da componente horizontal do gradiente geotérmico ser desprezível na ausência de fontes geotermais.

Conclui-se assim que o efeito termo-elétrico não é relevante para a geração de anomalias de PE em aquíferos fissurais em rochas pré-cambrianas estáveis.

\section{Efeito Eletroquímico}

Para gerar uma d.d.p. numa célula eletroquímica no meio geológico é necessário que águas de diferentes salinidades estejam separadas por uma formação argilosa. Esta formação deve ser espessa, impermeável e sem descontinuidades, para que não ocorra fluxo hidráulico apreciável que possa promover mistura e homogeneização da salinidade. Em perfurações petrolíferas esta situação é garantida pelo revestimento artificial do poço com um reboco de argila, que isola a lama de perfuração da água nativa da formação.

Por outro lado, é bastante provável que as fraturas em rochas cristalinas possam conter argila disseminada, que foi carreada pela próprio movimento da água, ou até mesmo argila resultante da decomposição da rocha. Esta argila disseminada poderia ser fonte importante de polarização induzida. Contudo, dificilmente esta argila poderia ser importante como fonte de PE de origem eletroquímica porque ela não promove a separação completa de duas massas de água com salinidades diferentes, de modo a formar uma célula eletroquímica. A argila disseminada poderia, contudo, diminuir a influência do fenômeno eletrocinético, uma vez que ela reduz a permeabilidade.

No caso dos aqüíferos do cristalino do Nordeste Brasileiro, existe a possibilidade de que as águas das unidades 2, 3 e 4 (Fig. 1) apresentem salinidades um pouco diferentes. Devido à melhor condição de lixiviação nas proximidades do riacho, é possível que a água contida na unidade 2 seja menos salina do que as águas contidas nas unidades 3 e 4 . Contudo, para gerar uma anomalia de PE, com amplitude de $10 \mathrm{mV}$ numa temperatura da ordem de $50{ }^{\circ} \mathrm{C}$, o contraste de resistividade das águas deve ser aproximadamente igual a 1,5, considerando-se o caso de soluções de cloreto de sódio (Schlumberger, 1972). A salinidade das águas das rochas cristalinas (em equivalente $\mathrm{NaCl}$ ) está, geralmente, no intervalo entre $500 \mathrm{mg} / \mathrm{l} \mathrm{e} 1.000 \mathrm{mg} / \mathrm{l}$. Admitindo que este intervalo é válido para a unidade 2 , a salinidade da unidade 3 estaria no intervalo entre $850 \mathrm{mg} / \mathrm{l} \mathrm{e} 1.500 \mathrm{mg} / \mathrm{l}$ (Schlumberger, 1972); ou seja, 


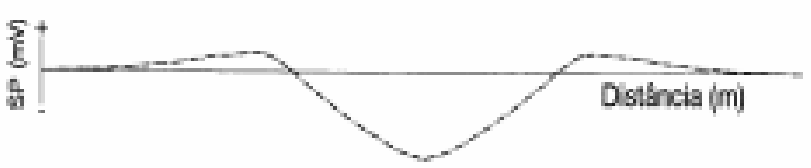

a) Anomalia de PE

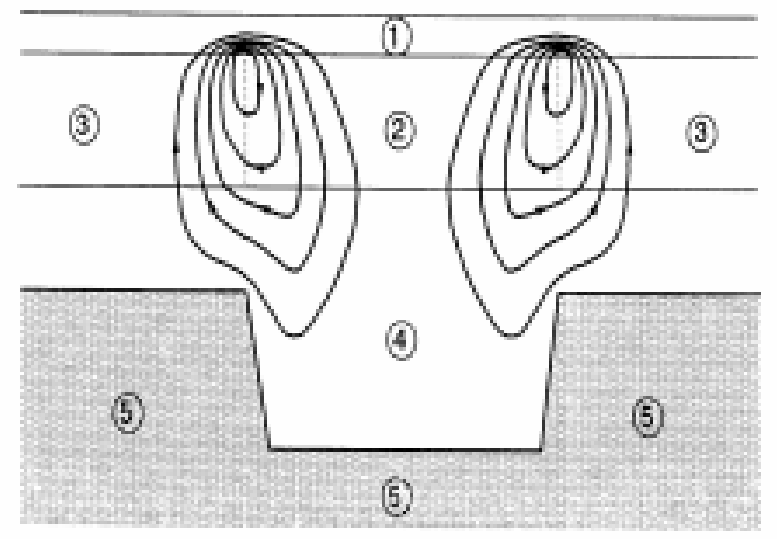

b) Padrão de circulaçâo de correntes

Fig. 2 - Padrão esquemático das células de corrente elétrica e do perfil de potencial espontâneo gerados no modelo de aqüífero.

Fig. 2 - Schematic pattern of the electric current cells and the self-potential profile associated with the aquifer model.

a salinidade da unidade 3 deveria ser, pelo menos, $50 \%$ maior que a da unidade 2. É bastante improvável que tal diferença de concentração possa ser mantida na ausência de uma barreira impermeável e, assim, é descartada a possibilidade de que células eletroquímicas possam constituir o mecanismo dominante de geração de PE em aqüíferos de fraturas.

Uma anomalia de PE pode ser formada sobre um corpo de sulfeto metálico quando este está numa profundidade tal que parte do mesmo se situa acima do nível freático (Sato \& Mooney, 1960). Embora não tenha sido investigada a presença ou não de corpos de sulfetos nos locais onde as perfilagens de PE - a serem apresentadas na próxima seção - foram realizadas, é improvável que estas anomalias possam estar associadas com corpos de sulfetos, face à multiplicidade de locais amostrados.

\section{Efeito Dominante}

Do exposto nas seções anteriores, conclui-se que, entre os efeitos analisados, o efeito eletrocinético é o mecanismo dominante de geração de anomalias de PE em aquífferos fissurais em rochas cristalinas.

\section{EXEMPLOS DE CAMPO}

Nesta seção, são apresentados perfis de PE obtidos em poços e na superfície do terreno em aqüíferos cristalinos de Itaberaba e Ipirá, Bahia. Será demonstrado que os dados de campo são consistentes com a conclusão de que o efeito eletrocinético é o mecanismo dominante de geração de anomalias de PE, bem como que perfis de $\mathrm{PE}$ podem ser úteis para otimizar a locação de poços em aquíferos fissurais em rochas cristalinas.

\section{Perfis de Poços}

Na Fig.3 são apresentadas perfilagens de PE e resistividade aparente $\left(\rho_{\mathrm{a}}\right)$ em três poços da Fazenda Bonita, Itaberaba (BA). O único tratamento efetuado, em cada perfil de PE, foi referenciar os valores de potencial elétrico a uma base de referência, situada na superfície do terreno. Os poços foram perfurados em locais próximos aos leitos de riachos (cerca de $10 \mathrm{~m}$ ), como é usual no Nordeste Brasileiro. Os três poços têm o mesmo perfil litológico, que é constituído por uma camada superficial areno-argilosa superposta à rocha cristalina, neste caso um granito-gnaisse. A camada superficial tem espessura de $8 \mathrm{~m}$ nos poços $\mathrm{P} 1 \mathrm{e}$ $\mathrm{P} 2$ e $2 \mathrm{~m}$ no poço $\mathrm{P} 4$, de acordo com as fichas dos poços descritas pela CERB (Companhia de Engenharia Rural da Bahia). Registre-se que a descrição destas fichas não permite inferir as zonas onde a rocha cristalina está fraturada.

Os perfis de $\rho_{\mathrm{a}}$ mostram, através de mínimos, a presença de zonas densamente fraturadas, a exemplo das anomalias em torno de $48 \mathrm{~m}$ e $60 \mathrm{~m}$ de profundidade no poço P-2. Em geral, estas anomalias de $\rho_{\mathrm{a}}$ estão correlacionadas com anomalias de PE. Nestas zonas, é que ocorre o fluxo de água entre as fraturas e o poço. Logo, a associação de anomalias de PE com zonas de entrada e saida de água das fraturas é consistente com a sua geração por efeito eletrocinético.

As perfilagens foram realizadas vários anos após a completação dos furos de modo que neles já havia ocorrido a homogeneização química das águas provenientes das várias fraturas e da camada de alteracão mais superficial. Apesar desta homogeneidade, observam-se anomalias de PE com amplitudes de $100 \mathrm{mV}$. Anomalias desta ordem de grandeza, e ocorrendo num fluido de salinidade aproximadamente uniforme, não 
podem ser explicadas por meio de células eletroquímicas. Registre-se que Soonawala (1983) mediu anomalias de PE de $175 \mathrm{mV}$ de amplitude em poços perfurados em granito, tendo também realizado perfilagens da condutividade da água presente no poço e verificado que esta era aproximadamente uniforme. Estes fatos são consistentes com a análise anterior, onde se indicou que o efeito eletrocinético é o mecanismo dominante de geração de anomalias de PE em aquiíferos cristalinos.

Não se dispõe de perfis de PE efetuados na superfície do terreno nos locais onde foram realizadas as perfilagens de poços. Caso tais perfis existissem, o par de perfis (poço + superfície) poderia, em cada local, ser referenciado a uma base comum, de modo que as polaridades das anomalias poderiam ser estudadas, vi-

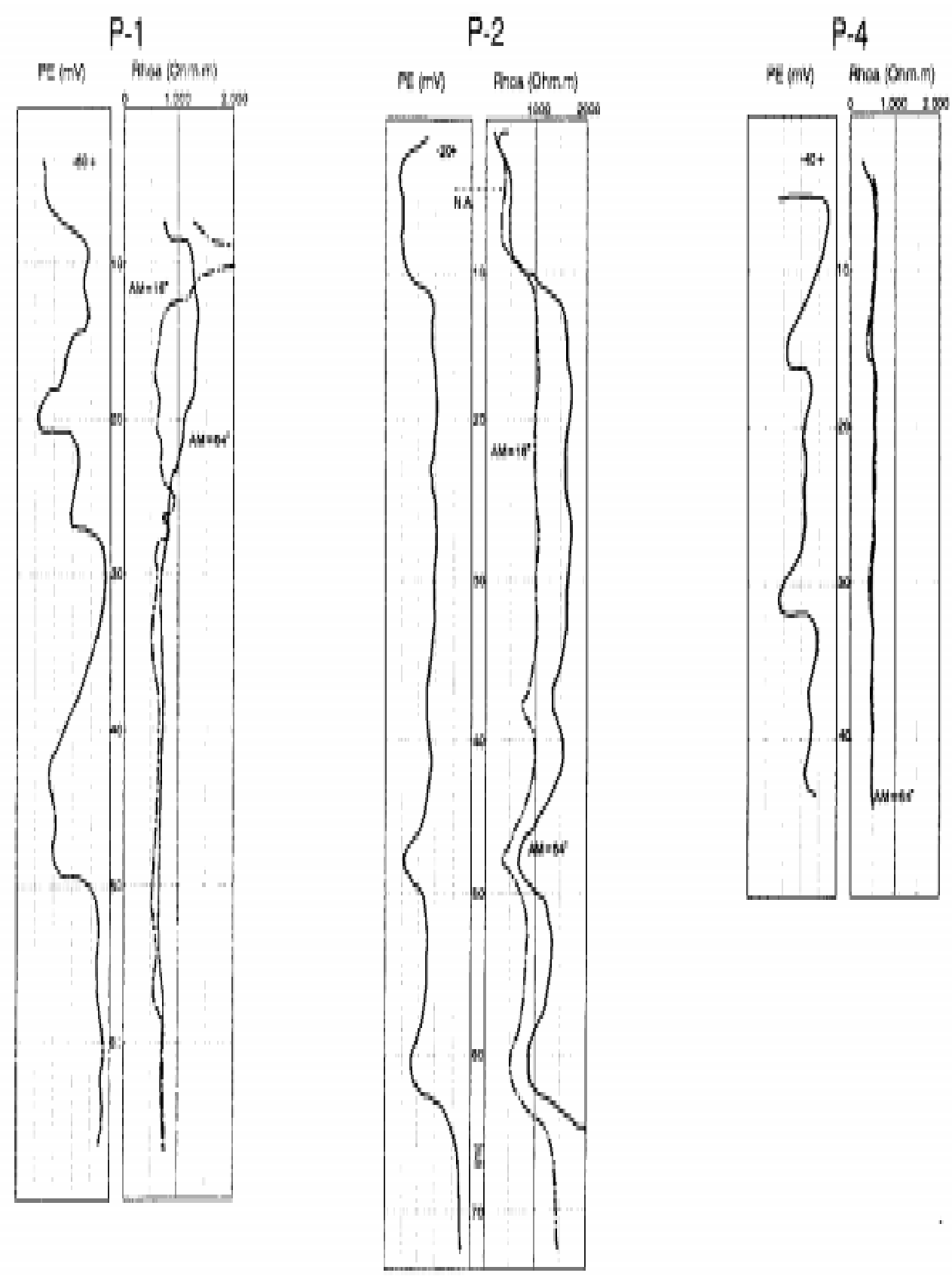

Fig. 3 - Perfis elétricos de três poços da Fazenda Bonita, Itaberaba (BA). Em cada poço, apresenta-se: na esquerda, o perfil de potencial espontâneo $(\mathrm{mV})$; no centro, a profundidade $(\mathrm{m})$ e, na direita, os perfis de resistividade aparente (Ohm.m) (curva contínua: arranjo normal longo, curva descontinua: arranjo normal curto).

Fig. 3 - Electric logs of three wells from Bonita Farm, Itaberaba (BA). For each well, it is shown: in the left portion, the selfpotential $\log (\mathrm{mV})$; in the central portion, the depth $(\mathrm{m})$; and, in the right portion, the apparent resistivity logs (Ohm.m) (long normal array: solid line, short normal array: dash line). 

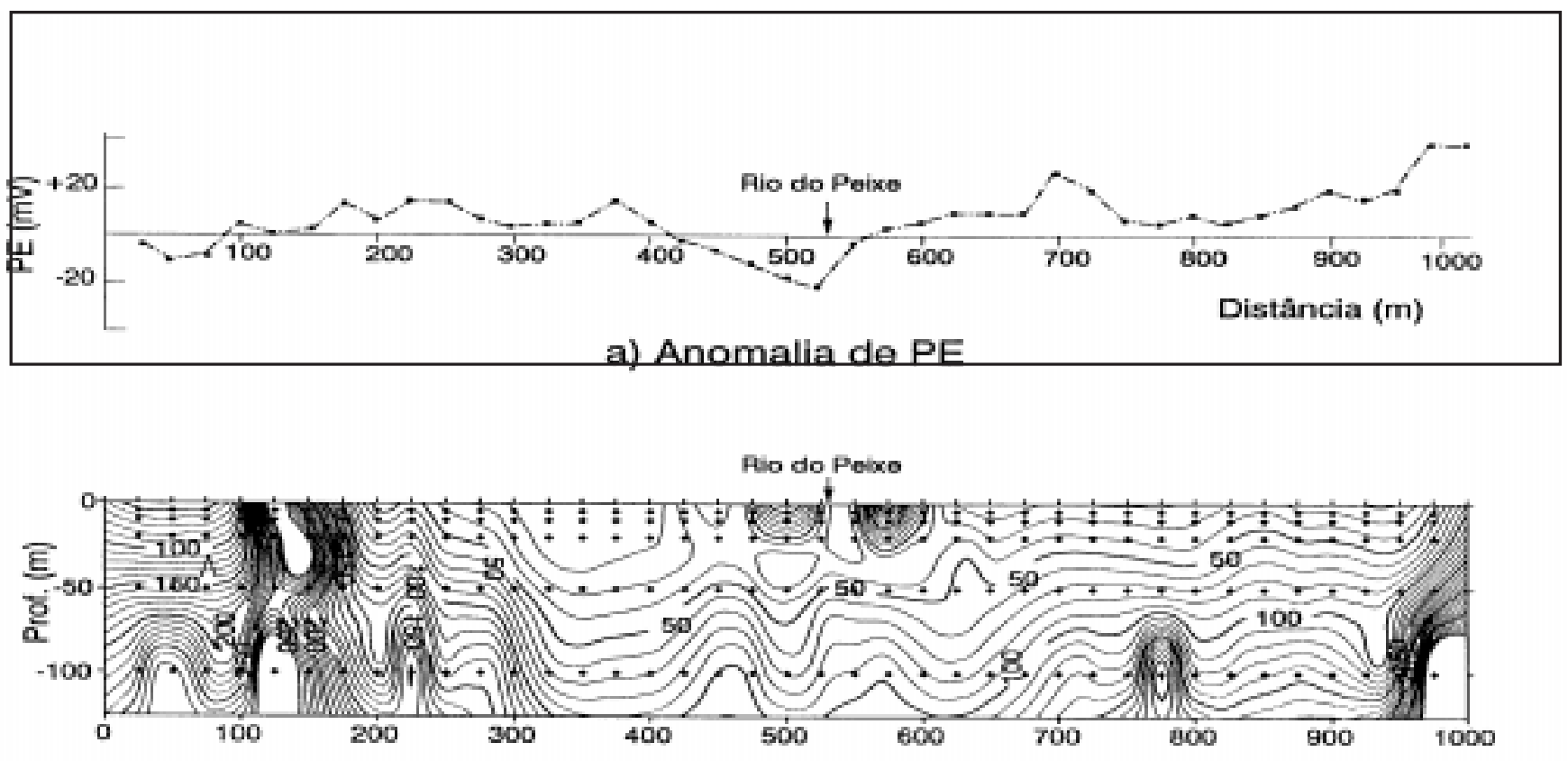

b) Seçâo de resistividade aparente

Figura 4 - Perfil de potencial espontâneo (a) e seção de resistividade aparente (b) - Ipirá (BA) - Exemplo 1.

Figure 4 - Observed self-potential profile (a) and pseudoresistivity section (b) - Example 1 from Ipirá (BA).

sando obter informações sobre o fluxo de água no aquífero. Contudo, mesmo na ausência de dados mais completos, observa-se que, em geral, as anomalias de PE mais profundas estão associadas com máximos (40 $\mathrm{m}-50 \mathrm{~m}$ em P-1, $48 \mathrm{~m}$ e $60 \mathrm{~m}$ em P-2, $15 \mathrm{~m}$ e $30 \mathrm{~m}$ em $\mathrm{P}-4)$ enquanto que existem mínimos em profundidades mais rasas (30 $\mathrm{m}$ em P-1, $8 \mathrm{~m}$ em P-4). No caso do poço $\mathrm{P} 2$, o perfil não mostra um mínimo próximo à superfície; contudo, este perfil mostra um forte gradiente de PE, em torno de $3 \mathrm{~m}$, que pode indicar um mínimo subsuperficial cuja existência não pode ser comprovada por causa da presença de um tubo de proteção do furo neste nível. Este padrão de polaridades é consistente com um padrão geral de fluxo da água subterrânea em que a trama de fraturas alimentaria o aluvião do leito do riacho, padrão este que é esperado para a estação não chuvosa e que é consistente com o modelo proposto (Fig. 1).

\section{Perfis Efetuados na Superfície do Terreno}

As Figs. 4, 5 e 6 mostram exemplos de campo de seções de $\rho_{\mathrm{a}}$ (arranjo Schlumberger) e perfis de PE levantados em aqüíferos fissurais em áreas de rochas cristalinas em Ipirá (BA). Os procedimentos de campo utilizados para levantar as seções de $\rho_{\mathrm{a}}$ estão descritos em Medeiros (1987) e também em Medeiros \& Lima (1990). A única diferença neste procedimento, em relação ao procedimento padrão de levantamento de sondagens elétricas, consiste na utilização de um sistema multi-cabo que permite fazer todas as medidas de $\rho_{\text {a }}$ sem efetuar mudanças de eletrodos. Por outro lado, nas medidas de PE utilizou-se o procedimento usual de amostrar os perfis por trechos (Anderson \& Johnson, 1976). Em cada trecho do perfil, são utilizados um eletrodo fixo e um eletrodo intinerante. Para cada posição do eletrodo intinerante, é medida a d.d.p. em relação ao eletrodo fixo. Utilizou-se um passo de afastamento do eletrodo intinerante, em relação ao eletrodo fixo, igual a $25 \mathrm{~m}$. A mudança de trecho é feita quando a relação sinal-ruído na medida de d.d.p. se deteriora. Posteriormente, todos os trechos do perfil são referenciados a uma única base. Para efetuar as medidas de PE, foram utilizados eletrodos porosos e uma solução de sulfato de cobre.

Os perfis de PE (Figs. 4a, 5a e 6a) mostram um padrão semelhante ao apresentado no perfil esquemático da Fig. 2a, com relação tanto à forma quanto à amplitude das anomalias. Em particular, observa-se que a semi-largura dos mínimos é de cerca de $100 \mathrm{~m}$. Desse modo, é bastante improvável que as fontes de PE estejam associadas apenas ao fluxo subterrâ- 


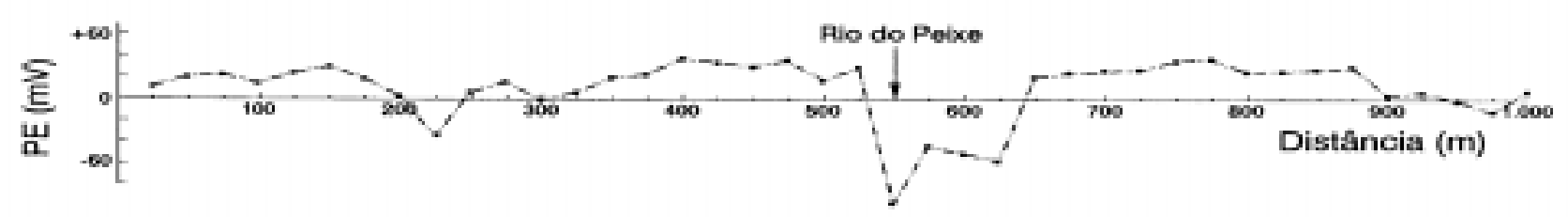

a) Anomalia de PE

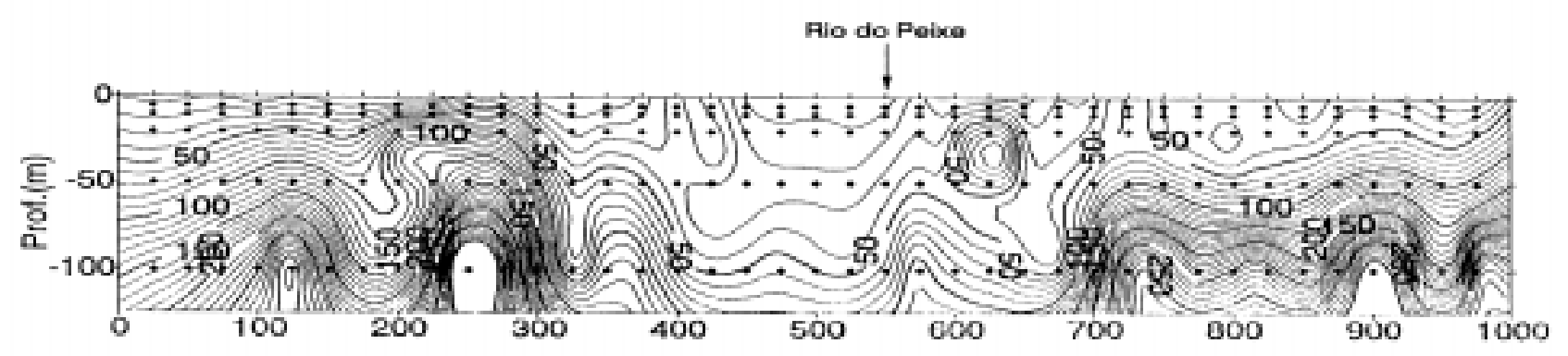

b) Seçāo de resistividade aparente

Figura 5 - Perfil de potencial espontâneo (a) e seção de resistividade aparente (b) - Ipirá (BA) - Exemplo 2.

Figure 5 - Observed self-potential profile (a) and pseudoresistivity section (b) - Example 2 from Ipirá (BA).

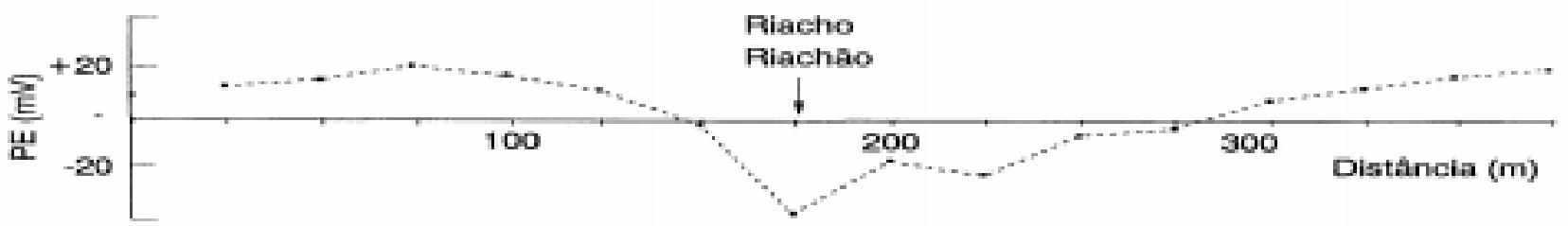

- NE

a) Anomalia de PE

SW

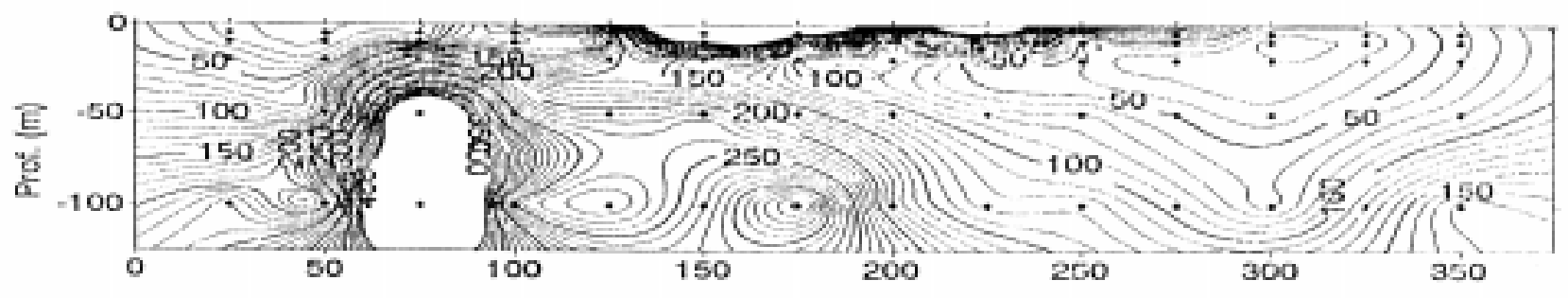

b) Seçāo de resistividade aparente

Figura 6 - Perfil de potencial espontâneo (a) e seção de resistividade aparente (b) - Ipirá (BA) - Exemplo 3.

Figure 6 - Observed self-potential profile (a) and pseudoresistivity section (b) - Example 3 from Ipirá (BA).

neo subsuperficial, nos aluviões dos riachos, pois estes têm larguras em torno de $10 \mathrm{~m}$.

As seções de $\rho_{\text {a }}$ fornecem boas estimativas das larguras e espessuras das zonas de alteração do cristalino, que estão associadas com as anomalias condutivas destas seções. Por exemplo, da análise da Fig. 5 b podese estimar que a zona de alteração e fraturamento do cristalino tem cerca de $200 \mathrm{~m}$ de largura (de $400 \mathrm{~m}$ a $600 \mathrm{~m}$ ) e que a espessura desta zona é cerca de $50 \mathrm{~m}$. Esta última estimativa se baseia na interpretação dos dados de $\rho_{\mathrm{a}}$, abaixo da estação $550 \mathrm{~m}$, usando o modelo de camadas planas paralelas. Contudo, as zonas de alteração podem ser localmente constituídas de rochas fraturadas ou aluvião preenchidos por água, que são 
de interesse para perfuração, mas podem também ser constituídas por material mais argiloso, que não é de interesse para perfuração. Por outro lado, os perfis de PE são dominantemente influenciados pelas variações nas condições de fluxo de água que estão fortemente associadas com as zonas de fraturas e/ou de constituição arenosa; as zonas de constituição argilosa atuam apenas como fontes de ruído nestes perfis. Exemplos de inferência da constituição dos produtos de alteração do cristalino pela análise comparativa das seções de $\rho_{\text {a }}$ e perfis de PE podem ser obtidos das Figs. 4, 5 e 6. Na Fig. 4, em torno da estação $350 \mathrm{~m}$, a seção de $\rho_{\mathrm{a}}$ apresenta uma forte anomalia condutiva, enquanto que o perfil de PE não apresenta nenhuma anomalia; estes fatos permitem inferir que a anomalia condutiva se deve a uma zona de constituição argilosa de alteração do cristalino. Inferência análoga pode ser feita comparando-se as Figs. 5a e 5b, em torno da estação 450 m, bem como comparando-se as Figs. 6a e 6b, em torno da estação 300 m.

A amplitude e a largura do mínimo do perfil de PE estão diretamente associadas com a importância do fluxo de água para a unidade 2 (Fig. 1). A largura desta unidade é aproximadamente igual à semi-largura do mínimo do perfil próximo do riacho. Além disso, as assimetrias em relação ao centro do riacho dos perfis de PE estão associadas com assimetrias nas variações das propriedades petrofísicas. Com base nestes elementos, uma análise comparativa de produtividade pode ser feita entre dois locais concorrentes para a locação de um poço. Por exemplo, pelo fato da amplitude do mínimo de PE da Fig. 5a ser o triplo do mínimo da Fig. 4a, pode-se concluir que a travessa associada à Fig. 5a é mais indicada para uma perfuração do que a associada à Fig. 4a. Além disso, a assimetria do perfil de PE da Fig. 5a revela que a borda SW do riacho, por estar associada a uma maior largura no perfil, é mais indicada para uma perfuração do que a borda NE. Com relação à Fig. 4a, a borda NE seria a mais indicada.

A análise integrada acima realizada constitui um acréscimo importante de informação em relação ao exame isolado das seções de $\rho_{\text {a }}$. Os exemplos apresentados revelam que a interpretação integrada das seções de $\rho_{\mathrm{a}}$ e perfis de PE pode não só indicar as zonas de alteração do cristalino mas também descartar, dentro destas zonas, setores que são de constituição argilosa e que não têm interesse para a locação de poços.

\section{CONCLUSÕES}

O mecanismo dominante de geração de anomalias de PE em aqüíferos fissurais em rochas cristalinas é o efeito eletrocinético associado ao fluxo hidrodinâmico natural do aquífero. Neste ambiente, os efeitos eletroquímico e termo-elétrico são mecanismos de geração secundários ou desprezíveis.

A interpretação integrada de seções de resistividade aparente e perfis de PE, levantados na direção transversal à direção dominante de fraturamento (conforme inferida pela presença de trechos retilíneos nos riachos), pode fornecer estimativas da largura e profundidade das zonas de alteração da rocha, bem como discriminar, dentro destas zonas, setores que são constituídos de rochas fraturadas e/ou de areias. Nestes setores, existe fluxo hidráulico relativamente importante que pode gerar fortes anomalias de PE. Esta associação de técnicas geo-elétricas pode se constituir numa excelente abordagem de exploração de detalhe de locais potencialmente favoráveis e concorrentes à locação de poços, que foram previamente selecionados com base na interpretação de fotografias aéreas.

\section{AGRADECIMENTOS}

Os autores agradecem ao Conselho de Desenvolvimento Científico e Tecnológico (CNPq) pelo auxílio financeiro fornecido na forma de bolsas de pesquisa. Os autores também agradecem aos revisores anônimos as sugestões e correções que muito contribuíram para melhorar a versão inicial do manuscrito.

\section{REFERENCIAS}

AHMAD, M.U. -1964- A laboratory study of streaming potentials. Geophys. Prosp., 12: 49-64.

ANDERSON, L.A. \& JOHNSON, G.R. -1976Applications of the self-potential method to geothermal exploration in Long Valley, California. Jour. Geophys. Res. 81: 1527-1532.

BENEDINI, M. -1976- The aquifers and their hidraulic characteristics. Geoexploration, 14: 157-178.

BOGOSLOVSKY, V.A. \& OLGIVY, A.A. -1972- The study of streaming potentials on fissured media models. Geophys. Prosp. 20: 109-117.

CORRY, C.E. -1985- Spontaneous polarization associated with porphyry sulphide mineralization. Geophysics, 50: 1020-1034.

CORWIN, R.F. \& HOOVER, D.B. -1979- The selfpotential method in geothermal exploration. Geophysics, 44: 226-245.

CORWIN, R.F. \& MORRISON, H.F. -1977- Selfpotentials variations preceding earthquakes in central 
California. Geophys. Res. Lett., 4: 171-174.

CORWIN, R.F., DEMOULLY, G.T., HARDING, R.S. \& MORRISON, H.F. -1981- Interpretation of selfpotential survey results from the East Mesa geothermal field. Jour. Geophys. Res., 86: 1841-1848.

DAKHNOV, V.N. -1962- Geophysical well loging. Colorado School of Mines Quart., 57(2), Golden, CO, USA, 445 pp.

DI MAIO, R. \& PATELLA, D. -1991- Basic theory of electrokinetic effects associated with earthquakes. Bolletino di Geofisica Teorica ed Applicata, 33: 145154.

DI MAIO, R. \& PATELLA, D. -1994- Self-potential anomaly generation in volcanic areas. Acta Vulcanologica, 4: 119-124.

DI MAIO, R., DI SEVO, V., GIAMMETTI, S., PATELLA, D., PISCITELLI, S. \& SILENZIARIO, C. -1996- Self-potential anomalies in some Italian volcanic areas. Annali di Geofisica, 37: 1137-1148.

FITTERMAN, D.V. -1978- Electrokinetic and magnetic anomalies associated with dilatant regions in a layered earth. Jour. Geophys. Res. 83: 5923-5928.

KELLER, G.V. \& FRISCHKNECHT, F.C. -1966Electrical methods in geophysical prospecting. Pergamon Press. Londres, England. 519 pp.

LIMA, O.A.L. \& MEDEIROS, W.E. -1988- Geophysical techniques applied to groundwater investigation of crystalline aquifers in central Bahia, Brazil. Exp. Abstr. of 58th Intern. Meet. Soc. Explor. Geophys., 1: 625628., Anaheim, CA, USA.

LOGN, O. \& BOLVIKEN, B. -1974- Self-potentials at the Joma pyrite deposit. Geoexploration, 12: 11-28.

MARSHALL, D.J. \& MADDEN, T.R. -1959- Induced polarization. A study of its causes. Geophysics, 24: 790796.

MEDEIROS, W.E. -1987- Eletro-resistividade aplicada à hidrogeologia do cristalino: um problema de modelamento bidimensional. Dissertação de Mestrado,
Universidade Federal da Bahia, 149 pp, Salvador, BA. MEDEIROS, W.E. \& LIMA, O.A.L. -1990- A geoelectrical investigation for groundwater in crystalline terrains of central Bahia, Brazil. Ground Water, 28: 518523.

MEDEIROS, W.E. \& LIMA, O.A.L. -1991- Potencial elétrico espontâneo em aquiúferos de fraturas: geração e uso hidrogeológico. Res. Exp. do 2o. Cong. Intern. Soc. Bras. Geofísica, 2: 1034-1038, Salvador, BA, Brasil.

MIZUTANI, H., ISHIDO, T., YOKOKURA, T. \& OHNISHI, S. -1976- Electrokinetic phenomena associated with earthquakes. Geophys. Res. Lett., 3: 365-368.

OLGIVY, A.A., AYED, M.A. \& BOGOSLOVSKY, V.A. -1969- Geophysical studies of water leakages from reservoirs. Geophys. Prosp., 22: 36-62.

PATELLA, D. -1997- Introduction to ground surface selfpotential tomography. Geophys. Prosp., 45: 653-681.

SATO, M. \& MOONEY, H.M. -1960- The electrochemical mechanism of sulfide self-potentials. Geophysics, 25: 226-249.

SCHIAVONE, D. \& QUARTO, R. -1984- Self-potential prospecting in the study of water movements. Geoexploration, 37: 47-58.

SCHLUMBERGER -1972- Log interpretation charts. Schlumberger Ltd., Houston, USA,92 pp.

SILL, W.R. -1983- Self-potential modeling from primary flows. Geophysics, 48: 76-86.

SOONAWALA, N.M. -1983- Geophysical logging in granites. Geoexploration, 21: 221-230.

WYNN, J.C. \& SHERWOOD, S.I. -1984- The selfpotential (SP) method: an inexpensive reconnaissance and archaeological mapping tool. Jour. of Field Archaeology, 11: 195-204.

Manuscript submitted April 8, 1998 Revised version accepted March 10, 1999

\section{NOTA SOBRE OS AUTORES NOTE ABOUT THE AUTHORS}

Walter E. Medeiros

Engenheiro Civil (UFRN, 1981), Mestre em Geofísica (UFBA, 1987) e Doutor em Geofísica (UFPA, 1993). Ele é professor do Departamento de Física da UFRN desde 1982 e bolsista-pesquisador do CNPq. A partir de 1995, ele tornou-se membro do Programa de Pesquisa e Pós-graduação em Geodinâmica e Geofísica da UFRN, onde tem orientado trabalhos de mestrado. Seus principais interesses são teoria da inversão com métodos potenciais e elétricos e aplicações em geofísica regional e prospecção geofísica. Ele publicou 08 artigos em revistas internacionais, 02 artigos em revistas nacionais e apresentou 31 trabalhos em congressos nacionais e internacionais. Ele é membro da Sociedade Brasileira de Geofísica e da Society of Exploration Geophysicists (EUA).
Olivar A. L. Lima

Geólogo (UFRJ, 1967), Mestre em Geociências (UFRJ, 1970), Doutor em Geofísica (1979) e realizou pesquisas de Pós-Doutorado na Universidade do Texas, Austin (1989 - 1990). Ele é professor do Centro de Pesquisas em Geofísica e Geologia (antigo PPPG) da Universidade Federal da Bahia desde 1979 e é bolsista pesquisador do CNPq. Ele orientou várias teses de pós-graduação a nível de mestrado e doutorado. Seus principais interesses de pesquisa são Propriedades Físicas das Rochas e Prospecção Geofísica. Ele publicou 10 artigos em revistas internacionais e nacionais e mais de 30 trabalhos em congressos nacionais e internacionais. Em 1988, ele recebeu o prêmio de Pesquisador do Ano da UFBA. Entre outras atividades administrativas e de acessoria, ele foi Chefe do Departamento de Geofísica, Coordenador Geral do PPPG, Secretário para Assuntos de Ensino da SBGf, Editor Associado da Revista Brasileira de Geofísica e Membro do Comitê Assessor de Geofísica do $\mathrm{CNPq}$ e do PADCT. Ele é membro da Sociedade Brasileira de Geofísica e da Society of Exploration Geophysicists (EUA). 


\section{SELF-POTENTIAL ANOMALY GENERATION IN FRACTURED CRYSTALLINE ROCKS AND ITS USE TO BOREHOLE LOCATION}

Self-potential electric (SP) measurements made in wells and at the surface along traverses crossing aquifers in fractured crystalline rocks in the state of Bahia, Brazil, revealed the presence of strong anomalies related to groundwater movements. As a result of several geo-electric investigations (Medeiros, 1987; Lima \& Medeiros, 1988; Medeiros \& Lima, 1990, 1991), a simple 2-D geometric-hydraulic model is proposed for the fractured aquifers (Fig. 1). From the base to the top, the unities in this model can be described as: (i) Unity 5 is the fresh crystalline rock. (ii) Unity 4 is the fractured crystalline rock. (iii) Unities 3 and 2 are the results of weathering, having unity 2 a higher quantity of sand than unity 3 because of the nearness of the brook to the unity 2 ; in this way it suffers a higher degree of leaching than unity 3 . Finally, unity 1 is the soil above the water table. The unities 2 and 4 constitute the main structures for groundwater storage. Unity 2 has primary porosity and unity 4 , secondary porosity (fissures). Tab. 1 shows typical values for electrical conductivity, electrokinetic coefficient, and permeability for the unities of the fractured aquifer model shown in Fig. 1.

Using the aquifer model (Fig. 1 and Tab. 1) and the basic equations for ionic concentration and fluid (or heat) coupled flows, it was found that electrokinetic effects developed along fracture walls can produce anomalies of amplitude comparable to those observed in the field. Estimates obtained for other coupled processes, such as the electrochemical and the thermo-electric effects, suggest that the electrokinetic effect is the most important component for setting up SP currents in fractured zones of crystalline rocks. A schematic view for the generated flow of electric current is shown in Fig. 2 with the SP profile measured at the surface.

Fig. 3 shows electric logs of SP and apparent resistivity $\left(\rho_{\mathrm{a}}\right)$ data measured in wells at Bonita Farm (Bahia state, Brazil). The lithologic log from top to base comprise a sand-clay layer and the crystalline rock (granitegneiss). The top unity has $8 \mathrm{~m}$ at wells $\mathrm{P} 1$ and $\mathrm{P} 2$ and $2 \mathrm{~m}$ at well P4. The $\rho_{\mathrm{a}}$ profiles detect the fractures as minima. These fractured horizons are correlated with SP anomalies. Because the groundwater flow is controlled by the fractures, this correlation corroborates the assertion that the electrokinetic effect is the source for SP anomalies. Observe that the boreholes do not have mud cakes so that the SP anomalies can not be explained by electrochemical effects.

Figs. 4, 5, and 6 show field data for pseudoresistivity sections (Schlumberger array) and SP profiles measured along traverses, on the Earth's surface, crossing fractured aquifers in crystalline rocks in Bahia state, Brazil. Field procedures to obtain the resistivity sections are described by Medeiros \& Lima (1990) and, to obtain the SP profiles, by Anderson \& Johnson (1976). SP profiles are similar to the schematic profile shown in Fig. 2. The amplitude and width of the minimum in the SP profile, near the brook, are related to the strength of the groundwater flow to unity 2 (Fig. 1). As a rule of thumb, the width of unity 2 can be taken is the half of the width of the SP anomaly minimum. Additionaly, asymmetric shapes of the SP minimum can reveal asymmetric variation of the physical properties inside the aquifer.

Using the above characterics of the SP profiles, a comparative analysis can be performed among different traverses in order to choose the best target for drilling to produce water. As an example, observe that the amplitude of the minimum of the SP profile in Fig. 5a is the triple of the amplitude of the minimum in Fig. 4a. In this way, the traverse associated with Fig. 5 is a better target for drilling than the traverse associated with Fig. 4. Focussing on Fig. 5a, observe that the SW side of the brook is a better place for drilling than the NE side because the SW side is associated with a larger width of the SP anomaly minimum.

The sections of $\rho_{\mathrm{a}}$ can give good estimates of the dimensions of the unities above the fresh rock because these dimensions are associated with the dimensions of the conductive anomalies in the sections. However, these unities can be composed either by fratured rock (or alluvium) saturated with water or by weathering products with high clay content. Only the first type of composition has hydrogeologic interest. Because a high clay content can prevent water movement and, consequently, the generation of SP anomalies by the electrokinetic effect, joint interpretation of $\rho_{\mathrm{a}}$ sections and SP profiles allows to distinguish areas of relative high permeability within fractured rocks. As an example, compare Figs. 4a and 4b, around station at $350 \mathrm{~m}$; the $\rho_{\mathrm{a}}$ section shows a strong anomaly but the SP profile does not show any anomaly. So, below the station at $350 \mathrm{~m}$, the unities have probably a high clay content. Similar conclusion can be obtained by comparing Figs. 5a and 5b, around station at $450 \mathrm{~m}$.

As conclusion, it is suggested that the SP method can be used as a good auxiliary method to the resistivity method to optimize borehole locations in fractured crystalline rocks. 